Arq. Bras. Med. Vet. Zootec., v.62, n.4, p.784-790, 2010

\title{
Desmopatia degenerativa em equinos: métodos de diagnóstico em animais vivos
}

[Equine degenerative desmopathy: diagnostic methods in live animals]

\author{
S.V. Schwarzbach ${ }^{1}$, G.E.S. Alves ${ }^{2}$, E.G. Mello ${ }^{2}$, M.G. Gomes ${ }^{2}$, M.X. Silva ${ }^{2}$ \\ ${ }^{1}$ Universidade Estadual do Centro-Oeste do Paraná \\ Rua Simeão Camargo Varella de Sá, 3 \\ 85040-080 - Guarapuava, PR \\ ${ }^{2}$ Escola de Veterinária - UFMG - Belo Horizonte, MG
}

\begin{abstract}
RESUMO
A desmopatia degenerativa (DD) possui caráter sistêmico e manifesta-se por acúmulos de proteoglicanos (PG) na matriz extracelular (MEC) de tecidos que contenham colágeno. Este estudo teve o objetivo de diagnosticar equinos suspeitos de serem acometidos por DD, em um plantel de animais de raça nacional, segundo o ângulo da articulação metatarsofalangiana (AMF) e a presença de acúmulos de PG em amostras de ligamento da nuca (LN). Analisaram-se 123 equinos clinicamente sadios e somente três $(2,7 \%)$ deles, segundo o ângulo $\mathrm{AMF} \leq 146^{\circ}$, foram considerados suspeitos. Não houve diferença significativa entre os grupos. Quinze éguas foram submetidas ao exame do ângulo da AMF e à biópsia do $\mathrm{LN}$, das quais sete $(47,7 \%)$ foram consideradas suspeitas, segundo ângulo da $\mathrm{AMF}$, enquanto seis $(40 \%)$ apresentaram acúmulos de PG. Foram encontrados acúmulos de PG em três (20\%) éguas não suspeitas. Um animal suspeito não apresentou alterações histológicas compatíveis de DD.
\end{abstract}

Palavras-chave: equino, desmopatia, ligamento, proteoglicanos

\begin{abstract}
The prevalence of degenerative desmopathy $(D D)$ was studied in equines of national breeds, according to the metatarsophalangeal joint angle (MPA) and the presence of accumulation of proteoglycans $(P G)$ in samples of nucal ligament (NL) from live animals, according to their age. One hundred twenty three clinically healthy horses were used. Only three (2.7\%), that had their angle rate $M P A \leq 146^{\circ}$ were considered suspect, with no significant difference between groups. Fifteen mares were subjected to examination of the angle of the MPA and biopsy of NL as well had reduction of the MPA angle, and six (40\%) showed accumulation of PG. Accumulation of PG was found in three (20\%) not suspected mares. A suspected animal showed no histological changes compatible to DD
\end{abstract}

Keywords: equine, desmopathy, ligament, proteoglycans

\section{INTRODUÇÃO}

A desmopatia degenerativa (DD), também denominada desmite degenerativa do ligamento suspensório, é considerada uma afecção de caráter sistêmico que pode acometer equinos de diferentes raças, entre elas Paso Peruano (Mero e Scarlett, 2005), Árabe, Quarto de Milha e Puro Sangue Inglês (Halper et al., 2006). A falta de informações e os sinais clínicos, semelhantes a outras afecções do sistema locomotor de equinos e de humanos, resultam em confusão e insegurança para a realização do diagnóstico.

$\mathrm{Na}$ maioria dos animais acometidos pela DD, os sinais clínicos manifestam-se após 15 anos de idade (Dyson et al., 1995; Gibson e Steel, 2002), frequentemente nos membros pélvicos, podendo acometer os quatro membros. Os sinais clínicos característicos são: aumento de volume no corpo

Recebido em 11 de fevereiro de 2009

Aceito em16 de julho de 2010

E-mail: silkever@yahoo.com.br

Apoio: FAPEMIG 
e ramos do ligamento suspensório (LS), claudicação, dor (Mero, 2006) e diminuição do ângulo dorsal da articulação metacarpo/tarsofalangiana (AMF) (Mero e Scarlett, 2005). No entanto, a avaliação da redução do ângulo da AMF é subjetiva, o que mostra a necessidade de estabelecer parâmetros considerados normais em um plantel de equinos, para, então, serem avaliadas a existência e a prevalência de animais suspeitos de DD.

A patogenia da DD é desconhecida, enquanto a prevenção e o tratamento são empíricos e quase sempre paliativos. A limitação de seu conhecimento torna seu diagnóstico pouco frequente e, ainda, predominantemente especulativo, com base no histórico, sinais clínicos e exame de ultrassom, confirmado por exame histológico de ligamentos colhidos de equinos após o óbito. Embora se constatem acúmulos de proteoglicanos (PG) na matriz extracelular (MEC) de amostras teciduais de animais acometidos (Halper et al., 2006), há necessidade de desenvolver técnicas para a realização do diagnóstico da DD em animais vivos.

Apesar da presença dos sinais clínicos da DD em animais de raças nacionais, como Brasileiro de Hipismo, Mangalarga Marchador e mestiços, entre outras, não foram encontrados dados de sua prevalência na literatura consultada.

Este estudo teve o objetivo de realizar diagnósticos em animais suspeitos de serem acometidos de DD, em um plantel de equinos de raça nacional, segundo o ângulo da articulação metatarsofalangiana (AMF) e a presença de acúmulos de PG em amostras do ligamento da nuca (LN) em animais vivos, estudados segundo a idade.

\section{MATERIAL E MÉTODOS}

Este estudo foi aprovado pelo CETEA $\mathrm{n}^{\circ}$ 174/2006. Foram utilizados 118 equinos clinicamente sadios da Polícia Militar de Minas Gerais (PMMG), 64 machos e 54 fêmeas, da raça Brasileiro de Hipismo e mestiços da raça, e cinco fêmeas da raça Mangalarga Marchador, da Escola de Veterinária da Universidade Federal de Minas Gerais (EV/UFMG), com idades entre dois e 26 anos, escore corporal variando de 2 a 4 , segundo Speirs (1997). Cento e oito animais da
PMMG mantidos em baia, onde receberam $6 \mathrm{~kg}$ de ração comercial com $12 \%$ de proteína bruta e feno de coastcross à vontade, mantinham um regime de trabalho de oito horas diárias de exercício, predominantemente ao passo, para policiamento em área urbana de Belo Horizonte. Este plantel foi avaliado quanto à prevalência de animais suspeitos de DD considerando somente $o$ ângulo da AMF. As 15 éguas restantes, dez da PMMG e cinco da EV/UFMG, mantidas em piquetes onde receberam $0,5 \mathrm{~kg} / 100 \mathrm{~kg} \mathrm{PV} / \mathrm{dia}$ de feno coastcross, foram avaliadas segundo ângulo da AMF e a presença de acúmulos de PG no LN. Todos os animais foram separados em três grupos conforme a idade: até 7 anos, de 8 a 12 anos e igual ou mais de 13 anos.

O manejo dos animais da PMMG é baseado na criação das éguas gestantes em piquetes, situados no município de Florestal, na região metropolitana de Belo Horizonte, local destinado à reprodução do plantel. Os potros são mantidos em liberdade, em piquetes, até atingirem a idade de dois anos, quando são levados para o quartel, em Belo Horizonte, para serem iniciados na doma e rotina de policiamento, de oito horas diárias.

Para a realização da morfometria angular, utilizou-se um artrogoniômetro (Carci Indústria e Comércio de Aparelhos Cirúrgios e Ortopédicos Ltda., São Paulo, SP) de $15 \mathrm{~cm}$ de comprimento, cujo eixo foi posicionado sobre o centro de movimento da AMF, enquanto suas hastes acompanhavam os raios ósseos do metatarso e falanges. Para a medida do ângulo cranial das articulações metatarsofalangianas, os animais foram mantidos em ambiente de piso plano e nivelado, com os membros paralelos em posição de estação forçada, como preconizado por Lage (2001).

Os animais foram considerados suspeitos quando apresentaram os ângulos da AMF, de ambos os membros pélvicos, iguais ou menores que $146^{\circ}$, medidos pelo artrogoniômetro. $\mathrm{O}$ ângulo de limite inferior, estabelecido em $146^{\circ}$, para determinação dos animais suspeitos de DD, foi estabelecido a partir da subtração do maior desvio-padrão calculado $\left(7^{\circ}\right)$ do menor valor angular médio encontrado $\left(155,6^{\circ}\right)$. Do valor resultante, foi subtraído o maior valor médio de diferença apresentado entre os membros posteriores $\left(2,7^{\circ}\right)$. O valor de $145,9^{\circ}$ foi 
aproximado a $146^{\circ}$, devido à marcação intervalada de 2 graus do artrogoniômetro.

Para a realização de biópsia do ligamento da nuca, as éguas foram colocadas em brete de contenção. Demarcou-se a área a $7 \mathrm{~cm}$ da borda cranial da escápula na região cervical, e realizaram-se tricotomia, antissepsia e anestesia local com cloridrato de lidocaína a 1\% (Hipolar Farmacêutica Ltda. Sabará, MG) por infiltração em $\mathrm{L}$ invertido de $3 \mathrm{~cm}$ de extensão, em ambos os lados. Após uma incisão vertical, de $1 \mathrm{~cm}$, com bisturi, da pele e do tecido subcutâneo, a cânula de biópsia 14G (Cânula de biópsia modelo Trucut- Biomedical- BM 1410, Delebrio, Itália), para tecidos moles, foi direcionada em ângulo reto ao LN e inserida aberta, a fim de obter fragmento de sentido transversal. A cânula penetrou o LN e foi fechada para a secção do fragmento. Seguindo-se à biópsia, realizou-se a dermorrafia, empregando-se sutura padrão simples separado com fio de náilon 2-0 (Monofilamento preto Nylon,Technofio) agulhado, que foi retirado 10 dias após.

As amostras foram processadas e incluídas em parafina segundo o método descrito por Luna (1968). Foram feitos cortes em micrótomo em secções de $5 \mu \mathrm{m}$, corados pelas técnicas de hematoxilina e eosina (HE), para análise morfológica, e de Alcian Blue (AB) pH 2,5, para evidenciar as PG. As secções transversais do LN foram avaliadas por microscopia de luz, em objetiva de imersão. A amostra de LN que apresentou maiores acúmulos de PG corou-se com mais intensidade pelo $\mathrm{AB} p \mathrm{pH} 2,5$ e foi utilizada como controle positivo para a classificação das demais, em positivas ou negativas, segundo a presença e o acúmulo de PG.

As médias dos ângulos das AMF dos membros direito e esquerdo de animais da mesma faixa etária foram submetidas à análise estatística não paramétrica, usando-se o teste Wilcoxon. Para a avaliação dos valores angulares de AMF conforme a faixa etária, foi utilizado o teste de Kruskall Wallis. O grau de significância estabelecido para os resultados foi de $5 \%$. Todas as análises foram realizadas no pacote Biostat 3.0 (Ayres et al., 2003).

\section{RESULTADOS E DISCUSSÃO}

Os resultados das mensurações dos ângulos articulares encontram-se na Tab. 1 .

O critério de inclusão, para suspeitos de DD, considerado neste estudo, quando ambos os membros pélvicos apresentam ângulo da $\mathrm{AMF} \leq 146^{\circ}$, diminui a possibilidade de inclusão de outras afecções do sistema locomotor, pois a característica sistêmica da DD contribui para a manifestação clínica em mais de um membro.

Embora houvesse semelhança entre as médias angulares dos grupos avaliados, três animais apresentaram ângulos de AMF de ambos os membros com valores menores ou iguais a $146^{\circ}$ (Tab. 1). A taxa dessa ocorrência equivale a $2,7 \%$ na população estudada, valor esperado, já que os animais estudados resultam de cruzamentos selecionados pelos veterinários da PMMG, objetivando parâmetros morfométricos adequados ao esporte. Adicionalmente, os animais avaliados foram mantidos sob o mesmo manejo e trabalho, e a influência da idade e a exigência do trabalho sobre o LS resultaram em respostas fisiológicas individuais, envolvendo mecanismos de degeneração ainda a serem estudados.

Mero e Scarlett (2005) e Mero (2006) observaram influência da idade na manifestação de DD, porém o reduzido número de animais suspeitos encontrados nesse estudo não apresenta relevância comprobatória da influência da idade na manifestação clínica da DD.

A baixa prevalência de animais suspeitos entre os 108 equinos avaliados somente pela mensuração do ângulo da AMF (Tab. 1) pode ser explicada com base na observação de Mero (2006), pois os sinais clássicos da DD, como a diminuição da AMF, ocorrem em menos de 50\% dos animais portadores de DD, mesmo em casos avançados. A observação de três animais com $\mathrm{AMF} \leq 146^{\circ}$, ainda que em taxa reduzida, indica a existência de animais suspeitos de DD no plantel estudado.

A análise de variância mostrou semelhança entre os ângulos da AMF direito e esquerdo dos animais do mesmo grupo e entre as médias das faixas etárias avaliadas, sem diferença significativa (Tab. 2). 
Desmopatia degenerativa em equinos...

Tabela 1. Medidas dos ângulos das articulações metatarsofalangianas de 108 equinos, machos e fêmeas, agrupados segundo a faixa etária

\begin{tabular}{|c|c|c|c|c|c|c|c|c|}
\hline \multicolumn{3}{|c|}{$\begin{array}{l}\text { Até } 7 \text { anos } \\
(\mathrm{n}=40)\end{array}$} & \multicolumn{3}{|c|}{$\begin{array}{c}\text { De } 8 \text { a } 12 \text { anos } \\
(n=37)\end{array}$} & \multicolumn{3}{|c|}{$\begin{array}{c}\geq 13 \text { anos } \\
(\mathrm{n}=31)\end{array}$} \\
\hline \multirow[t]{2}{*}{ Nome } & \multicolumn{2}{|c|}{ Ângulo } & \multirow[t]{2}{*}{ Nome } & \multicolumn{2}{|c|}{ Ângulo } & \multirow[t]{2}{*}{ Nome } & \multicolumn{2}{|c|}{ Ângulo } \\
\hline & Dir & Esq & & Dir & Esq & & Dir & Esq \\
\hline Soberano & 160 & 156 & Machê & 164 & 162 & Ironia & 154 & 160 \\
\hline Pimpdros & 158 & 146 & Nacional & 150 & 150 & Flauta & 154 & 154 \\
\hline Quatrim & 152 & 148 & Malino & 156 & 154 & Quitoco & 170 & 170 \\
\hline Quitanda & 158 & 160 & Picasso & 150 & 152 & Igaçaba & 156 & 156 \\
\hline Somali & 154 & 150 & Musgo & 160 & 160 & Homessa & 142 & 142 \\
\hline Palco & 144 & 160 & Outono & 140 & 146 & Cadete & 150 & 158 \\
\hline Palio & 142 & 156 & Pérola & 160 & 160 & Injúria & 136 & 134 \\
\hline Ribalta & 158 & 160 & Orquestra & 154 & 160 & Horizonte & 150 & 150 \\
\hline Serena & 156 & 150 & Micuim & 150 & 150 & Iliak & 150 & 152 \\
\hline Quilate & 152 & 150 & Life & 150 & 154 & Bacará & 150 & 150 \\
\hline Tagite & 156 & 150 & Liv & 150 & 148 & Farrapo & 154 & 152 \\
\hline Síria & 160 & 162 & Papoula & 162 & 160 & Impoluto & 152 & 156 \\
\hline Quantica & 156 & 160 & Panorama & 160 & 160 & Júpiter & 158 & 154 \\
\hline Quilha & 160 & 160 & Olimpo & 160 & 160 & Farol & 154 & 156 \\
\hline Ramadã & 168 & 164 & Orfeu & 154 & 156 & Garboso & 158 & 148 \\
\hline Reflexo & 160 & 160 & Haerofly & 168 & 160 & Hamburg & 152 & 150 \\
\hline Ritual & 150 & 150 & Larnac & 158 & 154 & Guarani & 160 & 158 \\
\hline Tafetá & 160 & 160 & Orvalho & 160 & 160 & Jequitibá & 160 & 160 \\
\hline Silício & 160 & 164 & Leviatã & 154 & 156 & Arapuã & 160 & 160 \\
\hline Turbina & 158 & 158 & Maltado & 160 & 160 & Lampião & 162 & 164 \\
\hline Tango & 160 & 160 & Opala & 160 & 156 & Heureca & 160 & 162 \\
\hline Turfa & 164 & 164 & Papaia & 158 & 158 & Luzeiro & 162 & 164 \\
\hline Totem & 162 & 160 & Primavera & 166 & 168 & Gran Cruz & 160 & 164 \\
\hline Serelepe & 160 & 162 & Néctar & 160 & 158 & Imperador & 162 & 160 \\
\hline Tela & 158 & 160 & Obelisco & 152 & 160 & Nióbio & 160 & 160 \\
\hline Turquesa & 158 & 154 & Montanha & 160 & 162 & Graciosa & 158 & 158 \\
\hline Taça & 160 & 160 & Lampejo & 160 & 160 & Gueto & 158 & 158 \\
\hline Tupã & 156 & 158 & Oriental & 164 & 168 & Minerva & 154 & 150 \\
\hline Tarô & 158 & 158 & Lusitano & 156 & 158 & Helena & 154 & 152 \\
\hline Tulipa & 158 & 160 & Orquídea & 160 & 158 & Impostor & 160 & 160 \\
\hline Tatuagem & 160 & 160 & Naia & 158 & 160 & Sagaz & 154 & 152 \\
\hline Resgate & 158 & 156 & Nirvana & 154 & 152 & & & \\
\hline Sueco & 162 & 160 & Líber & 158 & 158 & & & \\
\hline Qualidade & 156 & 160 & Passarela & 152 & 156 & & & \\
\hline Quiron & 160 & 162 & Onda & 150 & 156 & & & \\
\hline Ravel & 154 & 152 & Lança & 158 & 160 & & & \\
\hline Quevedo & 160 & 158 & Notável & 162 & 162 & & & \\
\hline Rena & 154 & 154 & & & & & & \\
\hline Queda & 158 & 150 & & & & & & \\
\hline Parcel & 166 & 166 & & & & & & \\
\hline
\end{tabular}

Dir: direito; Esq: esquerdo.

Os valores, médias, encontrados foram semelhantes aos observados por Silva (2006), em equinos atletas de alto desempenho de Concurso Completo de Equitação, de raças de origem europeia $(157,86 \pm 2,41)$, resultados já esperados, pois, além de terem sido avaliados animais de uma raça resultante de cruzamentos de equinos europeus, o manejo a que estavam submetidos os equinos da PMMG proporcionava liberdade de locomoção e movimentação voluntária dos potros mantidos em piquetes, o que, segundo Smith et al. (2002), promove desenvolvimento estrutural e fisiológico adequado dos componentes do sistema locomotor. 
Tabela 2. Médias e desvios-padrão (DP) do ângulo dorsal das articulações metatarsofalangianas e idade de 108 equinos agrupados por faixa etária. Valores mínimo e máximo do ângulo referido

\begin{tabular}{llllll}
\hline \multicolumn{7}{c}{ Medida angular } \\
\hline Grupo (faixa etária) & Direito & Esquerdo & Média+DP & Mínimo & Máximo \\
\hline Até $7(\mathrm{n}=40)$ & $157,6 \pm 4,8 \mathrm{Aa}$ & $157,4 \pm 5,0 \mathrm{Aa}$ & $157,5 \pm 4,9$ & 142,00 & 168,00 \\
8 a $12(\mathrm{n}=37)$ & $156,9 \pm 5,5 \mathrm{Aa}$ & $157,6 \pm 4,7 \mathrm{Aa}$ & $157,2 \pm 5,1$ & 140,00 & 168,00 \\
$\geq 13(\mathrm{n}=31)$ & $155,6 \pm 6,3 \mathrm{Aa}$ & $155,6 \pm 7,0 \mathrm{Aa}$ & $155,6 \pm 6,7$ & 134,00 & 170,00 \\
Total $(\mathrm{n}=108)$ & $156,8 \pm 5,6$ & $156,9 \pm 5,6$ & $156,9 \pm 5,6$ & 134,00 & 170,00 \\
\hline
\end{tabular}

Letras distintas, minúsculas na linha e maiúsculas na coluna, determinam diferença entre os valores $(\mathrm{P}<0,05)$.

A técnica de biópsia de LN, praticada nas éguas mantidas em piquetes, foi desenvolvida previamente em um piloto com três animais. $\mathrm{O}$ procedimento provou ser seguro, viável e minimamente invasivo para a colheita de amostras de LN, visando ao diagnóstico histológico da DD, conforme sugerido por Halper et al. (2006). Foi realizado sem a necessidade de administrar sedativos, com os animais contidos em brete e sob efeito de anestesia local por infiltração. Adicionalmente, outro aspecto que reforça essa classificação é a espessura da cânula de biópsia $14 \mathrm{G}$, semelhante a de uma agulha hipodérmica ou cateter utilizados na rotina clínica.

Não foi observada degeneração de fibras colágenas, neovascularização ou infiltrado inflamatório nas amostras de LN. Nas secções de
LN que não apresentaram acúmulos de PG, corados pelo método de $\mathrm{AB} \mathrm{pH} 2,5$, observou-se a coloração azulada, de distribuição homogênea, devido àa discreta distribuição de PG.

Algumas amostras de LN apresentaram aumento da basofilia, localizado entre as fibras de colágeno e septos, quando as secções de LN foram coradas em $\mathrm{HE}$, correspondentes ao acréscimo da intensidade azulada e à utilização do método de $\mathrm{AB} \mathrm{pH}$ 2,5. Considerando que o corante $\mathrm{AB}$ reage especificamente com os mucopolissacarídeos (Luna, 1968), o aumento da intensidade azulada, devido aos acúmulos de PG na MEC, permitiu a utilização do controle positivo para avaliação histológica das amostras de LN (animal marcado em negrito na Tab. 3) para animais acometidos de DD.

Tabela 3. Identificação, idade, ângulos dorsais das articulações metatarsofalangianas dos membros direito e esquerdo, de equinos suspeitos de desmite degenerativa do suspensório da articulação metatarsofalangiana e presença de acúmulos de proteoglicanos (PG) em 15 éguas

\begin{tabular}{|c|c|c|c|c|c|}
\hline \multirow[b]{2}{*}{ Nome } & \multirow[b]{2}{*}{ ID } & \multicolumn{2}{|c|}{ Ângulo } & \multirow[b]{2}{*}{ Suspeito } & \multirow[b]{2}{*}{ Acúmulos (PG) } \\
\hline & & Dir & Esq & & \\
\hline Nação & 9 & 154 & 150 & NÃO & NEGATIVO \\
\hline Palestina & 8 & 152 & 160 & NÃO & NEGATIVO \\
\hline Framboesa & 23 & 160 & 166 & NÃO & POSITIVO \\
\hline Faceira & 17 & 150 & 154 & NÃO & POSITIVO \\
\hline Fiesta & 17 & 142 & 142 & SIM & POSITIVO \\
\hline Sósia & 22 & 146 & 146 & SIM & POSITIVO \\
\hline Rendada & 22 & 114 & 110 & SIM & POSITIVO \\
\hline Razão & 5 & 145 & 140 & SIM & NEGATIVO \\
\hline Orgia & 7 & 145 & 140 & SIM & POSITIVO \\
\hline Caiana & 20 & 145 & 146 & SIM & POSITIVO \\
\hline Neve & 9 & 150 & 150 & NÃO & NEGATIVO \\
\hline Branq & 17 & 130 & 136 & SIM & POSITIVO \\
\hline Alfa & 10 & 142 & 150 & NÃO & NEGATIVO \\
\hline Troncha & 16 & 146 & 148 & NÃO & POSITIVO \\
\hline Pitica & 17 & 144 & 152 & NÃO & NEGATIVO \\
\hline
\end{tabular}

Dir: direita; Esq: esquerda; ID: idade em anos. 
Com base nessas características, observaram-se acúmulos de PG entre os feixes de colágeno em nove $(60 \%)$ das amostras de LN avaliadas, sendo seis $(40 \%)$ das éguas, que foram consideradas suspeitas para DD pelas mensurações dos ângulos dorsais da AMF, e três (20\%) de éguas consideradas isentas de sinais clínicos de DD. Somente um animal $(6,6 \%)$, considerado suspeito, não apresentou alterações histológicas condizentes à DD, enquanto cinco $(33,3 \%)$ equinos hígidos não apresentaram alterações histológicas de LN (Tab. 4).

Tabela 4. Amostras de equinos clinicamente suspeitos e hígidos classificados segundo presença ou ausência de acúmulos de proteoglicanos (PG) ao exame histopatológico do ligamento da nuca

\begin{tabular}{cccc} 
& \multicolumn{2}{c}{ Acúmulos de PG } & Total \\
\cline { 2 - 3 } Sinal Clínico & Presença & Ausência & $7(46,7 \%)$ \\
Suspeito & $6(40 \%)$ & $1(6,6 \%)$ & $8(53,3 \%)$ \\
Hígido & $3(20 \%)$ & $5(33,3 \%)$ & $15(100 \%)$ \\
\hline Total & $9(60 \%)$ & $6(40 \%)$ & \\
\hline
\end{tabular}

O critério baseado na presença de ângulos dorsais das AMF inferiores a $146^{\circ}$, como sinal clínico para a suspeita de DD, mostrou certa sensibilidade, com diferença significativa $(\mathrm{P}=0,04)$, visto que, de sete animais assim considerados, seis $(85,7 \%)$ possuíam acúmulos de PG nas secções histológicas das biópsias de LN. Estes resultados estão muito próximos dos descritos por Halper et al. (2006), que verificaram esses achados em $89,47 \%$ das amostras de LN de equinos com suspeita de DD. A correlação positiva encontrada entre o acúmulo de PG em amostras de LN e o sinal clínico de redução do ângulo dorsal da AMF comprova a natureza sistêmica da DD, como observado por Halper et al. (2006).

Por outro lado, é importante ressaltar a ocorrência de acúmulos de PG em amostras de três animais (20\%) com angulação dorsal das AMF acima de $146^{\circ}$, bem como a observação de um animal $(6,6 \%)$ considerado suspeito pela avaliação dos ângulos das AMF sem acúmulos de PG. Essas ocorrências significam que os achados histológicos nem sempre estão relacionados com a intensidade da manifestação clínica, o que está parcialmente de acordo com Halper et al. (2006), quando afirmaram que a intensidade dos achados histológicos de amostras de LS não se correlacionam diretamente com a manifestação clínica. Pode-se sugerir que estes resultados, atribuídos às alterações histológicas, precederam à alteração clínica, o que explica o fato de existirem animais clinicamente assintomáticos, como citado por Mero (2006). No entanto, a ausência de acúmulos de PG em uma amostra de ligamento não o exclui da possibilidade de ser portador da DD, pois o tamanho reduzido do fragmento de $\mathrm{LN}$ pode restringir a avaliação, conduzindo o examinador a emitir um resultado falso negativo.

O uso do artrogoniômetro para a medida do ângulo da AMF, realizado neste estudo, representa um método de triagem eficiente para a classificação de animais em suspeitos e clinicamente hígidos, desde que se tenha o padrão angular de cada raça, pois, além de se mostrar uma técnica versátil, possibilita a comparação dos valores com a média da população.

Por essas e outras razões, ficam claras as necessidades de mais pesquisas tendo como objeto o tecido ligamentar (Schwarzbach et al., 2008), uma vez que as informações na literatura sobre patofisiologia de desmopatias são escassas e quase sempre extrapoladas de trabalhos em tecido tendíneo.

\section{CONCLUSÕES}

A mensuração do ângulo dorsal da articulação metatarsofalangiana mostrou ser um método complementar importante para a avaliação clínica preliminar de animais suspeitos de DD, mas não deve ser utilizada isoladamente para a conclusão do diagnóstico. A técnica de biópsia do LN mostrou ser prática e viável, sem prejuízo ao animal, pois permitiu a realização do exame histológico em equinos vivos. A correlação positiva entre a presença de acúmulos de PG no LN e de animais clinicamente suspeitos aumenta a credibilidade da avaliação do ângulo da AMF, quando considerado o ângulo limite de $146^{\circ}$, entre animais suspeitos ou não de DD. 


\section{AGRADECIMENTOS}

Os autores agradecem o apoio da FAPEMIG por meio do Projeto APQ-3675-5.04/07.

\section{REFERÊNCIAS BIBLIOGRÁFICAS}

AYRES, M.; MAYRE, J.; SANTOS, A.S. Biostat 3.0, Aplicações estatísticas nas áreas das ciências biológicas e médicas. Belém: Sociedade Civil Mamirauá, 2003.

DYSON, S.; ARTHUR, R.M.; PALMER, S.E. et al. Suspensory ligament desmitis. Vet. Clin. N. Am.: Equine Pract., v.11, p.177-215, 1995.

GIBSON, K.T.; STEEL, C.M. Conditions of the suspensory ligament causing lameness in horses. Equine Vet. Edu., v.14, p.39-50, 2002.

HALPER, J.; BYOUNGJAE, K.; KHAN, A. et al. Degenerative suspensory ligament desmites as a systemic disorder characterized by proteoglycan accumulation. BMC Vet. Res., v.2, p.12, 2006.

LAGE, M.C. Caracterização morfométrica dos aprumos e do padrão de deslocamento de equinos da raça Mangalarga Marchador e suas associações com a qualidade da marcha. 2001. 113f. Tese (Doutorado) - Escola de Veterinária, Universidade Federal de Minas Gerais, Belo Horizonte.
LUNA, L.G. Manual of histologyc staining methods of the Arms Force Institute of Pathology. New York: McGraw Hill, 1968.

MERO, J.L. Diagnostic protocol for degenerative suspensory ligament desmites. 2006. Disponível em: <http://www. angelfire.com>. Acessado em: 12 ago. 2006.

MERO, J.L.; SCARLETT, J.M. Diagnostic criteria for degenerative suspensory ligament desmites in Peruvian Paso horses. J. Equine. Vet. Sci., v.25, p.224-228, 2005.

SCHWARZBACH，S.V.; PAGLIOSA， G.M.; ROSCOE, M.P. et al. Ligamento suspensório da articulação metacarpometatarsofalangianas nos equinos: aspectos evolutivos, anatômicos, histofisiológicos e das afecções. Cienc. Rural, v.38, p.1193-1198, 2008.

SILVA, E.A.G. Avaliação morfométrica e do desempenho de cavalos atletas de Concurso Completo de Equitação, 2006. 104f. Dissertação (Mestrado) - Escola de Veterinária, Universidade Federal de Minas Gerais, Belo Horizonte.

SMITH, R.K.W.; BIRCH, H.L.; GOODMAN, S. et al. The influence of aging and exercise on tendon growth and degeneration-hypotheses for the initiation and prevention of strain-induced tendinopathies. Comp. Bioch. Phys., v.133, p.1039-1050, 2002.

SPEIRS, V.C. Exame clínico de equinos. Porto Alegre: Artmed, 1997. 